\title{
Magnetic field induced optical variation in nanosize iron oxide fluid-particles
}

\author{
K.T. Wu ${ }^{\text {a,* }}$, Y.D. Yao ${ }^{\text {b }}$, G.N. Rao ${ }^{\text {b,c }}$, Y.L. Chen ${ }^{\text {a }}$, J.W. Chen ${ }^{\text {c }}$ \\ a Department of Physics, Fu Jen University, Taipei, Taiwan \\ ${ }^{\mathrm{b}}$ Institute of Physics, Academia Sinica, Taipei, Taiwan \\ ${ }^{\mathrm{c}}$ Department of Physics, National Taiwan University, Taipei, Taiwan
}

Available online 7 April 2005

\begin{abstract}
The transmission of light through magnetic fluid containing the magnetic nanoparticles with diameters of 7,9 and $12 \mathrm{~nm}$ have been investigated as a function of particle size and thickness of the films with wavelength between 400 and $750 \mathrm{~nm}$, and applied magnetic fields up to $275 \mathrm{Oe}$. It was found that the transmittance decreased with the increasing particle size as well as film thickness. The transmittance increases with increasing wavelength and saturates at $700 \mathrm{~nm}$ at film thickness of $10 \mu \mathrm{m}$ for all samples. The onset of transmittance shifts to higher wavelength with increasing film thickness. For a given film thickness, the transmittance increases with increasing magnetic field for films with particle size of 7 and $9 \mathrm{~nm}$, but decreases in the particle size of $12 \mathrm{~nm}$. Moreover, under a given magnetic field, the transmittance decreases exponentially with the increasing film thickness. The ordered structures of the magnetic columns were formed in the magnetic fluid films under the influence of the external magnetic field. These ordered structures are responsible for the significant field dependent on observed transmittance. The transmittance of the samples is inverse proportional to the area covered by the droplets of the magnetic fluid.
\end{abstract}

(c) 2005 Elsevier B.V. All rights reserved.

Keywords: Magnetic fluid; Transmittance; Nano-particles

\section{Introduction}

Nanoscale particles exhibiting a superparamagnetic behavior have been intensively studied recently due to the enhanced and novel applications in biomedical and diagnostic fields $[1,2]$.

\footnotetext{
* Corresponding author: Fax: +886229021038.

E-mail address: phys1001@mails.fju.edu.tw (K.T. Wu).
}

The optical, magnetic, chemical and mechanical properties of nanoparticles depend mostly on the particle shape, size, composition and external magnetic field. Recently there has been considerable interest in magneto optical devices which combine magnetic and optical phenomena [3,4]. The magnetic field and wavelength dependence of the optical transmittance for some magnetic fluids have been reported [5-8]. In this paper, the variation 
of the transmission of light through the $\mathrm{Fe}_{3} \mathrm{O}_{4}$ magnetic fluid has been investigated as a function of external magnetic field and incident optical wavelength with different particle sizes as well as film thickness.

\section{Experimentals}

The synthesis of magnetite nanoparticles has been prepared by chemical co-precipitation method. The mean particle size is monitored by varying the $\mathrm{pH}$ value. The mean size strongly depends on the acidity, and decreases with increasing $\mathrm{pH}$ value. The magnetic fluids are made through dispersion of synthesized nanoparticles of $\mathrm{Fe}_{3} \mathrm{O}_{4}$ with oleic acid coating in kerosene. The crystal structure of the nanoparticles is the same as that of the bulk $\mathrm{Fe}_{3} \mathrm{O}_{4}$ from X-ray diffraction. The average particle size, shape and morphology were examined using transmission electron microscope (TEM). The TEM images reveal a uniform spherical shape of the particles with an average diameter of 7,9, and $12 \mathrm{~nm}$ and the size distribution can be fitted by log-normal function [9]. A drop of magnetic fluid is injected into glass cells to form a magnetic fluid film, and placed between the poles of an electromagnet and normal to the axis of light. The transmittance was measured as a function of incident wavelength between 400 and $750 \mathrm{~nm}$ and applied magnetic fields up to 275 Oe for all the films.

\section{Results and discussions}

Fig. 1 shows the variation of the transmittance $(T)$ as a function of wavelength in zero magnetic field for particle size of $12 \mathrm{~nm}$ with different film thicknesses of 10, 100, 500 and $1000 \mu \mathrm{m}$. In general, the transmission threshold shifts to lower energy and a reduction of the average transmission was observed with increasing magnetic fluid film thickness. This can be ascribed to the elongation of the traveling path as the light passes the films.

In the case of $10-\mu \mathrm{m}$ film thickness, the transmittance increases dramatically from zero near

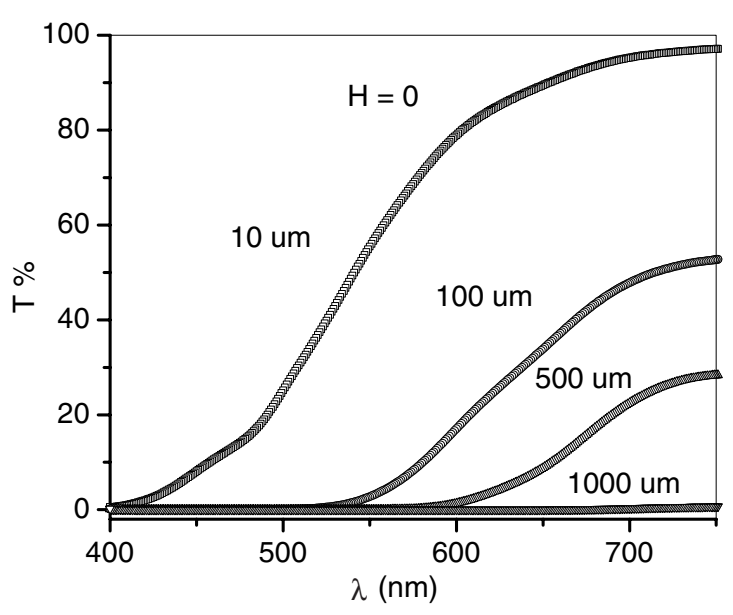

Fig. 1. The variation of the transmittance $(T)$ as a function of wavelength in zero magnetic field for particle size of $12 \mathrm{~nm}$ with different film thickness of 10, 100, 500 and $1000 \mu \mathrm{m}$.

wavelength $\lambda=400 \mathrm{~nm}$ to value of $97 \%$ at $\lambda=750 \mathrm{~nm}$. Similar behavior was also found with particle size of 9 and $7 \mathrm{~nm}$. The magnetic field dependent transmittance with incident wavelength of $750 \mathrm{~nm}$ for all the samples is depicted in Fig. 2. The transmittance increases rapidly in low magnetic fields for films with particle size of 9 and $7 \mathrm{~nm}$. This is due to the homogeneous separation and spherical shape of the nano-particles. The variation of transmittance due to magnetic field is very small in $10 \mu \mathrm{m}$ thick film (Fig. 2(a)) and maximum in $100 \mu \mathrm{m}$ thick film (Fig. 2(b)) for all the samples. It was found that, for a given film thickness, the transmittance increases with increasing magnetic field for films with 9 and $7 \mathrm{~nm}$ particles size, but it decreases for films with $12 \mathrm{~nm}$ particle size. This indicates that under an applied magnetic filed, the variation of the transmittance is mainly dependent on the area covered by the droplets of the emulsions in the magnetic fluid films.

To clarify the mechanism of these different behaviors, we studied their microscopic pictures. Fig. 3 shows the microscopic photographs of the ferrofluid under different perpendicular magnetic fields of: (a) 0.0 ; (b) 25 ; (c) 100 ; (d) 275 Oe for 9 nm particle size. In general, the dispersed magnetic particles agglomerate in perpendicular magnetic fields and the size of the droplet in the emulsions decreases with increasing magnetic field. 

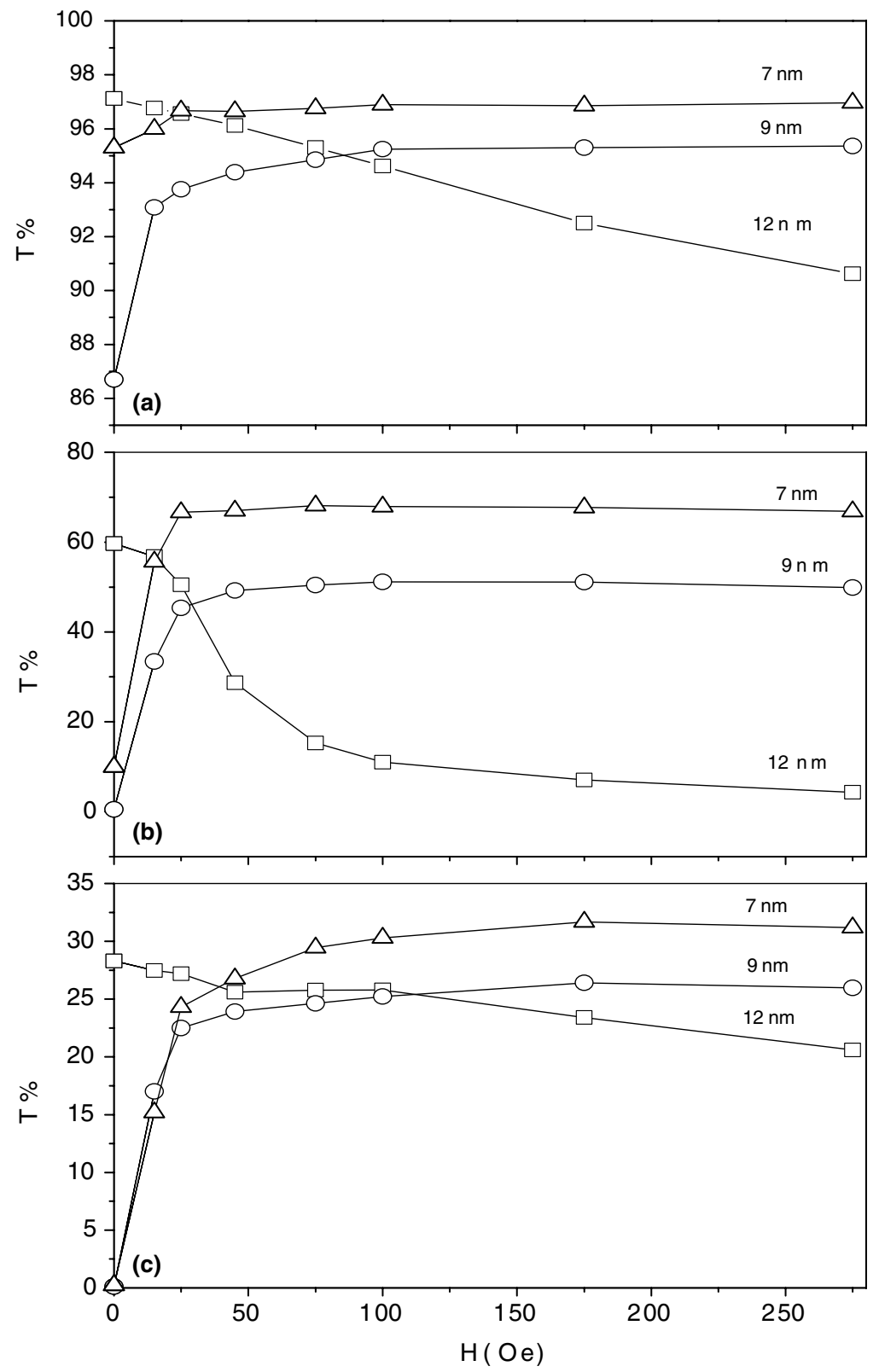

Fig. 2. The variation of the transmittance $(T)$ as a function of magnetic field with incident wavelength of $750 \mathrm{~nm}$ for all samples with film thickness of: (a) $10 \mu \mathrm{m}$; (b) $100 \mu \mathrm{m}$; (c) $500 \mu \mathrm{m}$.

The area covered by the droplets of the magnetic fluid calculating from the photographs and transmittance as a function of magnetic fields are depicted in Fig. 4 for the same $100 \mu \mathrm{m}$ film thickness with different particle sizes of 9 and $12 \mathrm{~nm}$.
The area covered by the droplets per unit area increases with increasing magnetic field for film of $12 \mathrm{~nm}$ particle size, where as it decreases for film of $9 \mathrm{~nm}$ particle size. From the above results, we conclude that the variation of transmittance is 


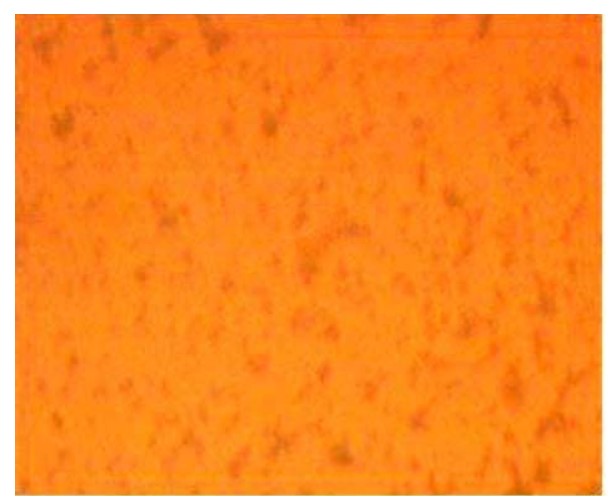

(a) $\mathrm{H}=0$

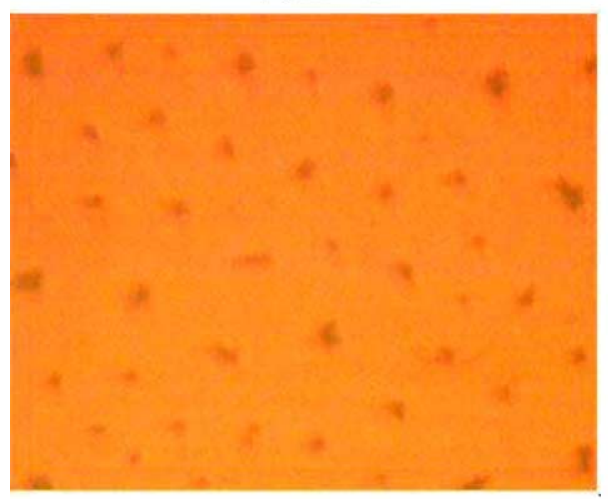

(c) $\mathrm{H}=100 \mathrm{Oe}$

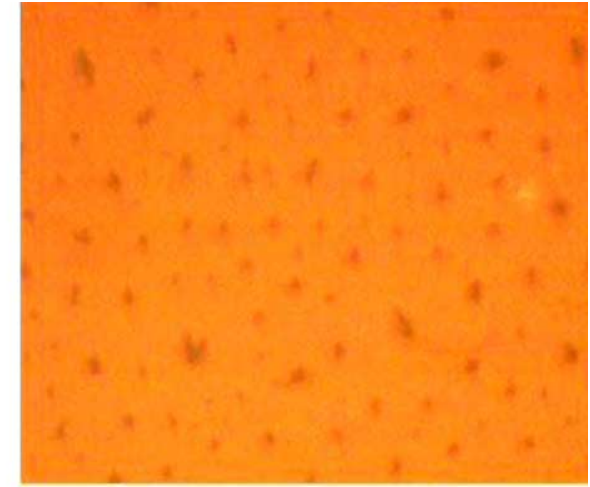

(b) $\mathrm{H}=25 \mathrm{Oe}$

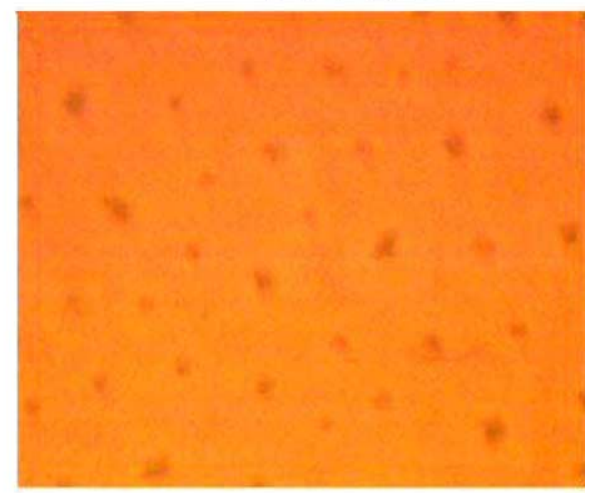

(d) $\mathrm{H}=275 \mathrm{Oe}$

Fig. 3. Optical micrographs of magnetic fluid with particle size of $9 \mathrm{~nm}$ at different perpendicular magnetic fields: (a) $H=0$; (b) $H=25$; (c) $H=100$; (d) $H=275$ Oe.

inverse proportional to the area covered by the droplets of the magnetic fluid. This different aggregation ability of the magnetic fluid particle is responsible for the variation of the optical transmittance under different magnetic fields.

The optical properties of nano-particle systems are closely related to the Mie resonance. This presents the prospect of observing new optical properties of nanoparticles as now the electric fields connected to the plasmon resonance (enhancement of the incoming and outgoing electric fields when interacting with light) will influence the effective fields around the neighboring nano-particles. The relationship between the particle size, shape and its spectrum of plasmon resonance absorption can be solved using the Mie and the Maxwell-Garnett theories $[10,11]$. In Mie theory, the interaction of spherical particles with light is treated by solving
Maxwell's equations with the boundary conditions for tangent components of the electric and magnetic fields at a spherical surface. Apell and Ljungbert [12] pointed out that the size dependent of the small particle plasmon resonance should be similar to wave vector dispersion of the flat surface plasmon. The surface plasmon absorption red shifts with increasing particle size. Thus, the optical absorption spectrum of plasmon resonance depends explicitly on the particle size [10-12].

While the clusters composed of simple nanoparticles, we could expect some similarities between the collective electronic excitations for surface and clusters. Moreover, it is found that the theory developed for the plane surface gives a good fit to the observed cluster size dependence of the Mie plasmon. In Fig. 1, the increasing film thickness corresponds to larger cluster size. Thus, the 


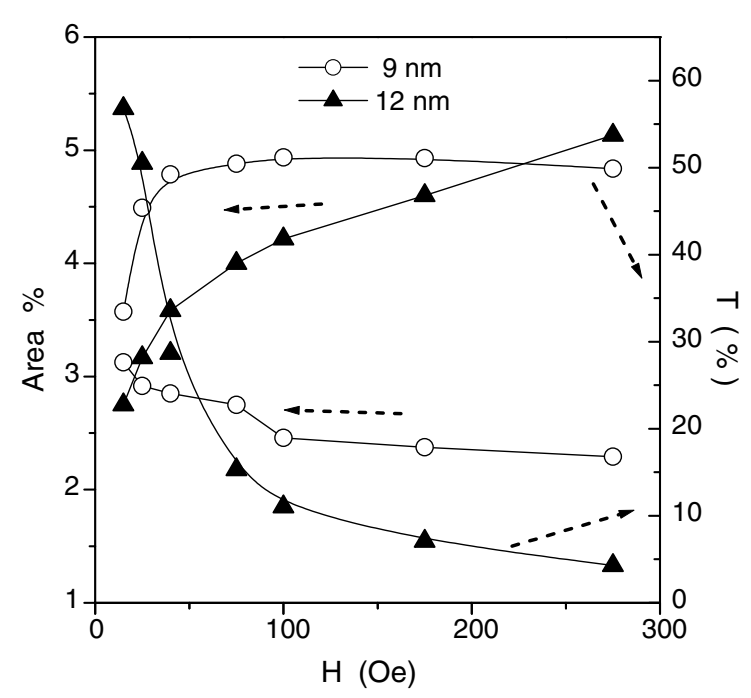

Fig. 4. The transmittance $(T)$ and the area covered (area \%) by the droplet as a function of magnetic field for $100 \mu \mathrm{m}$ films thickness with different particles size of 12 and $9 \mathrm{~nm}$.

plasmon absorption red shifts with increasing cluster size and the absorption peaks at longer wavelength. Therefore, the cut-off of transmittance shifts to longer wavelength with increasing film thickness as shown in Fig. 1.

While size and environment effects are very important, shape effects seem to be even more pronounced in the optical absorption spectrum of nano-particles. The plasmon resonance absorption band at lower energy, longer wavelength, is caused by the oscillation of the free electron along the major (long) rod axis and is known as the longitudinal surface plasmon absorption. This longitudinal plasmon absorption band maximum red shifts as the particles become more elongated along the major (long) axis of rodshaped. In the case of particle aggregation occurs, the plasmon resonance red shifts and often a second absorption peak at a longer wavelength is observed [10,11]. This band could be regarded as a longitudinal resonance absorption similar to the nanorods in the case of chain-like aggregation of the individual nano-particles. In Fig. 2, when the applied magnetic field is greater than $100 \mathrm{Oe}$, the agglomeration of dispersed magnetic nanoparticles becomes much more chain-like elongated along the perpendicular magnetic fields. The elongation increases significantly with increasing magnetic field for particle size of $12 \mathrm{~nm}$. And this would cause the longitudinal resonance absorption red shifts to longer wavelength. Therefore, at the incident long wavelength of $750 \mathrm{~nm}$, the absorption increases and the transmittance decreases with increasing magnetic field for particle size of $12 \mathrm{~nm}$. However, for smaller particle sizes of 7 and $9 \mathrm{~nm}$, the elongation along the magnetic field is insufficient to contribute the longitudinal resonance absorption to red shift. The transmittance is, therefore, nearly unchanged with increasing magnetic field greater than 100 Oe for particle sizes of 7 and $9 \mathrm{~nm}$ as shown in Fig. 2.

In general, our data of the optical properties of this nano-particle system are closely related and consistent with the Mie resonance. The particle size is a key factor in enabling the study of size-dependent physical properties of the nano-particles. The easy of turning the optical properties gradually with particle size and shape have made nanoparticles very interesting.

\section{Conclusions}

Magnetic nanoparticles with diameters of 7, 9, and $12 \mathrm{~nm}$ have been prepared by chemical co-precipitation method. The transmission of light through magnetic fluid containing these nanoparticles has been investigated as a function of particle size and thickness of the films with wavelength between 400 and $750 \mathrm{~nm}$, and applied magnetic fields up to 275 Oe. It was found that the transmittance decreased with the increasing particle size as well as film thickness. For a given film thickness, the transmittance increases with increasing magnetic field for films with particle size of 7 and $9 \mathrm{~nm}$, but decreases in the particle size of $12 \mathrm{~nm}$. The area covered by the droplets per unit area increases with increasing magnetic field for films of $12 \mathrm{~nm}$ particle size, where as it decreases for $9 \mathrm{~nm}$ particle size. So, the variation of transmittance is inverse proportional to the area covered by the droplets of the magnetic fluid. Our data are consistent with the Mie resonance. 


\section{Acknowledgment}

This work is supported by the Sapintia Education Foundation.

\section{References}

[1] B. Bonnemain, J. Drug Target. 6 (1998) 167.

[2] U. Hafeli, W. Schutt, J. Teller, M. Zborowski, Scientific and Clinic Applications of Magnetic Carriers, Plenum Press, New York, 1997.

[3] W. Luo, T. Du, J. Huang, Phys. Rev. Lett. 82 (1999) 4134.

[4] J.W. Seo, S.J. Park, K.O. Jang, J. Appl. Phys. 85 (1999) 5956.
[5] N. Inaba, H. Miyajima, H. Takahashi, S. Taketomi, S. Chikazumi, IEEE Trans. Magn. 25 (1989) 3866.

[6] K.T. Wu, Y.D. Yao, J. Magn. Magn. Mater. 201 (1999) 186.

[7] K.T. Wu, Y.D. Yao, H.K. Huang, J. Appl. Phys. 87 (2000) 6932.

[8] K.T. Wu, Y.D. Yao, T.C. Wu, Physica B 327 (2003) 319.

[9] C.G. Granqvist, R.H. Buhrman, J. Appl. Phys. 47 (1976) 2200.

[10] U. Kreibig, M. Vollmer, Optical Properties of Metal Clusters, Springer, Berlin, 1995.

[11] S. Link, M.A. El-Sayed, Int. Rev. Phys. Chem. 19 (2000) 409.

[12] P. Apell, A. Ljungbert, Solid State Commun. 44 (1982) 1367. 